\title{
Intracytoplasmic basophilic bodies in leukocytes in an HIV patient with pleural and pericardial effusion led to unexpected diagnosis
}

\author{
Hector Mesa $^{1,2}{ }^{(1)} \cdot$ Kristine Hummel $^{1} \cdot$ Susan Dachel $^{1} \cdot$ Juan C. Manivel $^{1}$
}

Received: 2 May 2017 / Accepted: 3 July 2017 / Published online: 12 July 2017

(C) US Government (outside the USA) 2017

\begin{abstract}
Human immunodeficiency virus (HIV) infections are declining worldwide; however, there are still $>1.2$ million HIV-infected people in the USA and $\sim 6700$ deaths/year can be directly attributed to HIV-related diseases. Opportunistic infections, Kaposi sarcoma, and non-Hodgkin lymphomas are always high in the differential diagnosis of HIV patients with systemic symptoms; however, causes of morbidity and mortality in HIV patients on highly active antiretroviral therapy are now approaching those of the general population. We present a case of an HIV patient who presented with B symptoms and body effusions. Diagnostic work-up geared towards infectious and neoplastic diseases led to the identification of intra- and extracellular rounded basophilic bodies in bone marrow and pleural effusion that were recognized as LE cells and allowed the diagnosis of systemic lupus erythematosus (SLE). We discuss the historical aspects of LE cell discovery, recent advances about their nature, ramifications of concurrent HIV-infection, and SLE and provide reference images of a finding that was originally described in bone marrow biopsies, but that nowadays has been nearly forgotten due to the development of alternative diagnostic tests.
\end{abstract}

Keywords HIV · LE cells · Systemic lupus erythematosus · Antinuclear antibodies $\cdot$ Caspases

Hector Mesa

Hector.Mesa@va.gov

1 Department of Laboratory Medicine and Pathology, Minneapolis Veteran Affairs Health Care System, Minneapolis, MN, USA

2 Minneapolis VA-Health Care System, Office BB-104, One Veterans Drive, Minneapolis, MN 55417, USA

\section{Introduction}

Despite a continuous decline in the incidence of human immunodeficiency virus (HIV) infections in the last decades thanks to effective public health measures and highly active antiretroviral therapy (HAART), there are more than 1.2 million HIV-infected people in the USA and $\sim 6700$ deaths per year can be directly attributed to HIV-related diseases $[1,2]$. Opportunistic infections, Kaposi sarcoma, and non-Hodgkin lymphomas are always high in the differential diagnoses of HIV patients with systemic symptoms; however, it is necessary to acknowledge that in HIV patients with access to HAART the causes of morbidity and mortality are now approaching those of the general population [3]. We present a case of an HIV patient who presented with B symptoms and body effusions. A diagnostic work-up focused in excluding infections and lymphoma led to the identification of intra- and extracellular rounded basophilic bodies in bone marrow and pleural effusion. Their identification prompted specific immunologic work-up and the diagnosis of systemic lupus erythematosus (SLE).

\section{Clinical history}

A 41-year-old male with previously asymptomatic HIV infection on HAART presented with several months of quantified night fevers $\left(103{ }^{\circ} \mathrm{F} / 39.4{ }^{\circ} \mathrm{C}\right)$, night sweats, poor appetite, $6 \mathrm{lb} / 2.7 \mathrm{~kg}$ weight loss, pleuritic chest pain, and migrating abdominal pains. He had consulted various providers several times and received various therapies without improvement. Chest computerized tomography (CT) performed at one visit showed pleural effusions and possible pulmonary infiltrates; he received albuterol and amoxicillin-clavulanate for possible bronchospasm and pneumonia. A CT-angiogram was 
negative for embolic disease. The patient returned due to lack of improvement and a repeat CT showed a mild pericardial effusion; an echocardiogram confirmed this finding, but was otherwise unremarkable. He was prescribed colchicine and ibuprofen for presumed pericarditis. Later, he consulted an outside center for a flare of his chest pain. Laboratory chemistry including cardiac enzymes did not show abnormalities. An electrocardiogram showed sinus rhythm initially, but later atrial flutter for which he was started on diltiazem. He also received hydromorphone, fentanyl, and lorazepam for his pain and anxiety and was transferred to our hospital for admission. Initial evaluation showed unremarkable vital signs, physical and neurologic exam; laboratory work-up showed a recent decline in CD4 count to $<200$ cells/mm3, increased LDH $203 \mathrm{U} / \mathrm{L}$ (range 87-24), and increased acute phase reactants: ESR $51 \mathrm{~mm} / \mathrm{h}$ (range 5-15), CRP $104 \mathrm{mg} / \mathrm{L}$ (range 0-3), ferritin $421.8 \mathrm{ng} / \mathrm{mL}$ (range 22-322). Repeat CT of chest/ abdomen and pelvis confirmed pleural and pericardial effusion and slight hepatosplenomegaly. An internal medicine and infectious disease evaluation included a negative HIV viral load, negative blood, urine and stool cultures, negative Histoplasma antigen and QuantiFERON®-TB Gold test, negative stool PCR for enteropathogenic bacteria and viruses. However, since disseminated fungal infection and body cavity lymphoma were still considered possible diagnoses, pleural effusion fluid tap, fine needle aspiration (FNA) of an axillary lymph node and a bone marrow biopsy were performed. The node FNA did not show abnormalities. The bone marrow biopsy buffy coats and pleural fluid cytospin showed lupus erythematosus cells (LE-cells). Subsequent rheumatologic work-up showed high homogeneous pattern antinuclear antibody (ANA 1:640 range: $<1: 40$ ) and anti-double stranded DNA (anti-dsDNA; 1:320 range: $<1: 10$ ) titers. During his hospital stay the patient also developed bilateral hand pain and swelling, and joint tenderness, fulfilling diagnostic criteria for the diagnosis of systemic lupus erythematosus (SLE): serositis, arthritis, ANA+, anti-dsDNA+. Rheumatology started the patient on prednisone and hydroxychloroquine with substantial improvement in symptoms that allowed his discharge.

\section{Material and methods}

A bone marrow (BM) aspirate, thoracentesis, and ultrasoundguided FNA specimens were submitted for morphologic, flow cytometric, and microbiology studies. Morphologic evaluation included Wright \& Giemsa (W\&G) stained smears of peripheral blood (PB), direct, buffy-coat/concentrate and touch imprints of BM aspirate and biopsy, Feulgen stained concentrate smears, and hematoxylin \& eosin (H\&E) stained clot and core biopsy specimens; diff \& quick (D\&Q) and Papstained smears of the axillary node FNA and direct, ThinPrep pap smear, W\&G cytospin preparation and $\mathrm{H} \& \mathrm{E}$ stained cellblocks of pleural effusion; microorganism stains: GomoriMethenamine-Silver (GMS) and Acid-Fast-Bacillus (AFB) stains were performed in BM clot \& core biopsy and pleural effusion cell block. Immunohistochemical stains for CD3 (Thermo Fisher Scientific, Fremont, CA, USA) and CD20 (Novacastra, Newcastle upon Tyne, UK) were performed in the BM biopsy. Flow cytometry of all three specimens was performed on a 6-colors BD FACSCanto ${ }^{\mathrm{TM}}$ analyzer (BD Biosciences, San Jose, CA, USA). All procedures were performed in compliance with institutional guidelines (Veterans Health Administration handbook 1200.05).

\section{Results}

Buffy coat preparations of BM and cytospin of pleural fluid showed neutrophils (PMN) and histiocytes with intracellular basophilic rounded bodies with a ground glass appearance; the BM concentrate also showed extracellular rounded bodies surrounded by neutrophils and amorphous clusters of basophilic material (Fig. 1). The intracellular bodies in the pleural specimen showed various stages of disintegration (Fig. 2). Notably, these changes were absent in the direct, touch imprints and ThinPrep preparations. The rounded bodies were negative for GMS stain for fungi and positive with Feulgen stain, indicating they contained DNA and represented degenerated nuclei. The remaining microorganism stains were negative and immunophenotypic studies excluded lymphoma. The findings were interpreted as LE cells.

\section{Discussion}

LE cells are PMN with phagocytosed, variably degenerated nuclei. They were originally described in $25 \mathrm{BM}$ specimens of patients with confirmed or suspected SLE by Dr. Malcom M. Hargraves and his team at the Mayo Clinic (Rochester, MN, USA) in 1948 [4]. Their description also included the presence of extracellular rounded and amorphous masses of basophilic material shown to be degenerated nuclei by the Feulgen stain, which stains specifically nucleic acids. This observation was seminal, as the subsequent discovery that this phenomenon also occurred in buffy coats of PB and BM specimens of normal individuals after incubation with serum of SLE patients led to the discovery of autoantibodies/autoimmunity, and spurred efforts for developing other specific tests for the diagnosis of rheumatologic diseases [5]. LE cells have been described in all body fluids and were considered a diagnostic criterion for SLE until the 2012 revision of the American College of Rheumatology classification criteria by the Systemic Lupus International Collaborating Clinics group $[5,6]$; however, most immunology/hematology laboratories 
Fig. 1 Buffy coat bone marrow aspirate. a-c The preparations show intracellular (arrow), extracellular (chevron), and amorphous conglomerates (arrowhead) of extruded nuclei partially degraded by antinuclear antibodies; notice the contrast with mechanically damaged naked and smudge nuclei (pentagon) (Wright Giemsa, $\times 100$ objective). d Feulgen stain demonstrates that the round bodies are nuclei (objective $\times 100$ )

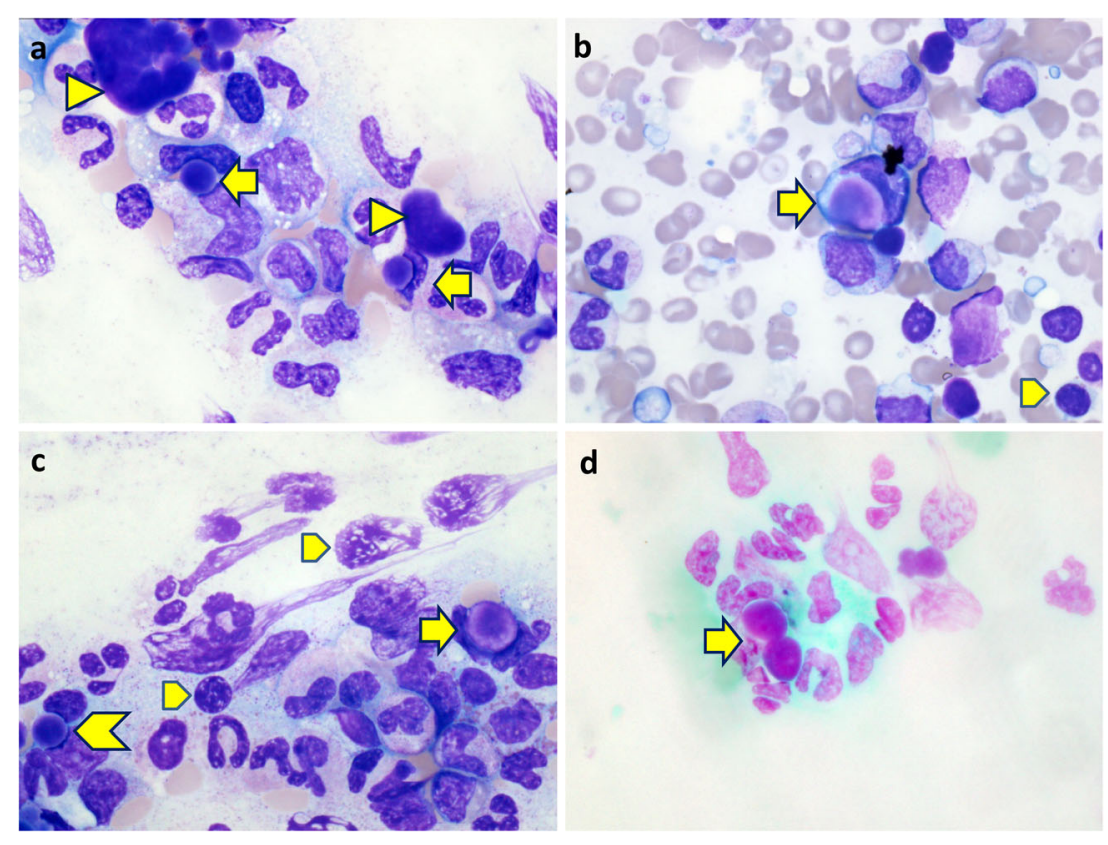

had stopped performing the LE-cell test long before that, due to the advent of less cumbersome and more sensitive and specific tests. These events account for the lack of familiarity of newer generations of hematology technicians and hematopathologists with this highly specific phenomenon, even when LE cells were described in BM biopsy specimens and were previously considered essential for the diagnosis of SLE [7]. So, why are LE cells primarily observed in buffy coat/cytospin preparations? The answer is that the centrifugation step of these preparations causes mechanical disruption of cells and allows incubation of the extruded nuclei with the patient's serum and remaining viable leukocytes, allowing their opsonization and phagocytosis [8]. It is also possible that the spontaneous degeneration that occurs in longstanding or delayed processing of fluid samples may contribute to this phenomenon. Why is the phagocytosis preferentially mediated by PMN and less frequently by macrophages? Experimental studies have shown that anti-dsDNA antibodies slowdown the decay of nuclei of damaged cells, which in turn preferentially activate PMN [9]. Why do extruded nuclei from SLE patients appear so different from the intact naked or smudged nuclei commonly observed in smears and from
Fig. 2 Buffy coat pleural fluid. Classic LE cells (arrow) and histiocytes (arrowhead) with variably digested nuclei (Wright Giemsa, $\times 100$ objective)

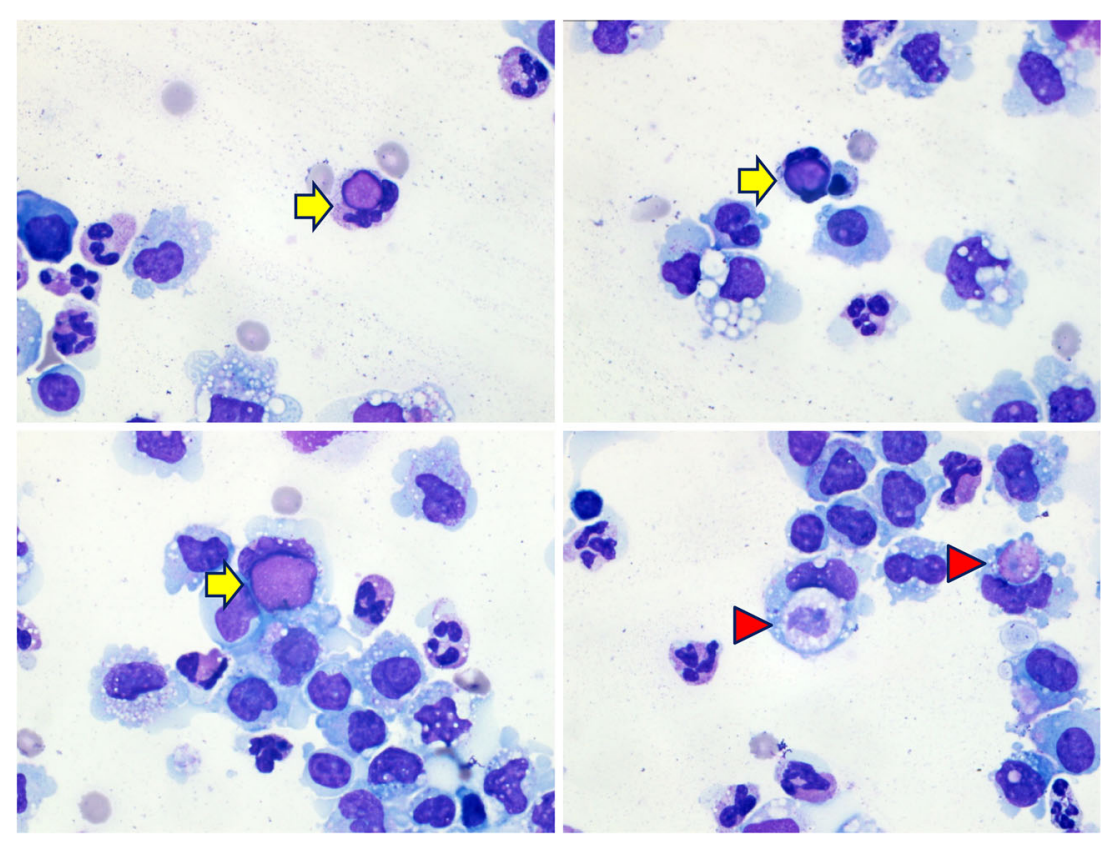


apoptotic cells with pyknotic, fragmented nuclei? In contrast to cell damage during the smearing process, which is purely mechanical, the extruded nuclei of SLE patients have been affected by the action of ANA [10]. Independent studies have shown that anti-dsDNA antibodies prevent the caspasemediated cleavage of nuclei through the Fas/Fas ligand-pathway, which is the normal mechanism triggered by DNA damage that leads to their rapid decay during programmed cell death. This abnormal alternative pathway causes persistence of damaged DNA and is likely responsible for the unusual ground-glass/amorphous appearance of degenerate nuclei in LE cells $[9,10]$. Since both HIV infection and SLE cause dysregulation of the immune response, is there an association between HIV and SLE? Studies on the subject [11, 12] report that treatment of SLE with immunosuppresants may lead to emergence of an unknown HIV infection, on the other hand, HAART may lead to de-novo or reactivation of SLE after immune reconstitution in HIV patients. SLE-associated autoantibodies appear to have a protective effect against HIV infections, and HIV-immunosuppression leads to decreased SLE-activity, so that the concurrence of both disorders is lower than expected according to their individual prevalence, but not mutually exclusive.

Take-home message from this case: causes of morbidity and mortality in HIV patients on HAART are now approaching those of the general population; concurrent SLE and HIV-infection is lower than expected by their individual prevalence, but not mutually exclusive; LE cells are usually found in buffy coat/cytospin preparations of body fluids, PB and BM biopsies; the unusual appearance of extruded and phagocytized nuclei in SLE patients is the result of inhibition of the normal apoptosis caspase mediated cleavage of damaged DNA by anti-dsDNA antibodies; anti-dsDNA antibodies prevent nuclear decay and favor phagocytosis by PMN over macrophages; recognizing LE cells and ANA induced nuclear changes is nowadays a nearly lost skill by hematology technicians and hematopathologists, because BM biopsies and LE cell test are now considered obsolete for the diagnosis of SLE.
Compliance with ethical standards The manuscript, or parts of it, have not been and will not be submitted elsewhere for publication. All authors have read and approved the manuscript. All authors acknowledge substantial participation and responsibility for this work. The authors do not have conflicts of interest or funding sources to declare. All procedures were performed in compliance with institutional guidelines (Veterans Health Administration handbook 1200.05).

\section{References}

1. Centers for Disease Control and Prevention (2015). HIV surveillance report. Vol 27

2. Kochanek KD, Murphy SL, Xu J, Tejada-Vera B (2016) Deaths: final data for 2014. Natl Vital Stat Rep 65(4)

3. Smith CJ, Ryom L, Weber R et al (2014) Trends in underlying causes of death in people with HIV from 1999 to 2011 (D:A:D): a multicohort collaboration. Lancet 384(9939):241-248

4. Hargraves MM, Richmond H, Morton R (1948) Presentation of two bone marrow elements; the 'tart' cell and 'LE' cell. Mayo Clin Proc 23:25-28

5. Hepburn AL (2001) The LE cell. Rheumatology 40(7):826-827

6. Petri M, Orbai AM, Alarcón GS et al (2012) Derivation and validation of systemic lupus international collaborating clinics classification criteria for systemic lupus erythematosus. Arthritis Rheum 64(8):2677-2686

7. Haserick JR, Sundberg RD (1948) The bone marrow as a diagnostic aid in acute disseminated lupus erythematosus. J Invest Dermatol 11:209-213

8. Lachmann PJ (1961) A two-stage indirect L.E. cell test. Immunology 4:142-152

9. Böhm I (2004) LE cell phenomenon: nuclear IgG deposits inhibit enzymatic cleavage of the nucleus of damaged cells and support its phagocytic clearance by PMN. Biomed Pharmacother 58(3):196201

10. Schmidt-Acevedo S, Pérez-Romano B, Ruiz-Argüelles A (2000) 'LE cells' result from phagocytosis of apoptotic bodies induced by antinuclear antibodies. J Autoimmun 15(1):15-20

11. Barthel HR, Wallace DJ (1993) False-positive human immunodeficiency virus testing in patients with lupus erythematosus. Semin Arthritis Rheum 23:1-7

12. Mody GM, Patel N, Budhoo A, Dubula T (2014) Concomitant systemic lupus erythematosus and HIV: case series and literature review. Semin Arthritis Rheum 44(2):186-194 